\title{
Internal Audit as a Quality Assurance Tool to Ensure Staff and Patient Safety During COVID-19 Pandemic
}

\author{
Suneeta Dubey ${ }^{1, *}$, Shalinder Sabherwal ${ }^{2}$, Javed Hussain Farooqui ${ }^{3}$, Prerna Garg ${ }^{4}$, Umang Mathur $^{3}$, \\ Ashok Kumar Singh ${ }^{5}$ \\ ${ }^{1}$ Quality Assurance, Glaucoma Services, Dr. Shroff's Charity Eye Hospital, New Delhi, India \\ ${ }^{2}$ Community Ophthalmology and Public Health Research, Dr. Shroff's Charity Eye Hospital, New Delhi, India \\ ${ }^{3}$ Cornea, Cataract and Refractive Surgery, Dr. Shroff's Charity Eye Hospital, New Delhi, India \\ ${ }^{4}$ Dr. Shroff's Charity Eye Hospital, New Delhi, India \\ ${ }^{5}$ Quality Assurance, Dr. Shroff's Charity Eye Hospital, New Delhi, India
}

Email address:

dubeysuneeta@hotmail.com (S. Dubey), shalinder.sabherwal@sceh.net (S. Sabherwal),jhfarooqui@gmail.com (J. H. Farooqui), prer.11@gmail.com (P. Garg), umang@sceh.net (U. Mathur)

${ }^{*}$ Corresponding author

\section{To cite this article:}

Suneeta Dubey, Shalinder Sabherwal, Javed Hussain Farooqui, Prerna Garg, Umang Mathur, Ashok Kumar Singh. Internal Audit as a Quality Assurance Tool to Ensure Staff and Patient Safety During COVID-19 Pandemic. International Journal of Ophthalmology \& Visual Science. Vol. 6, No. 3, 2021, pp. 158-163. doi: 10.11648/j.ijovs.20210603.12

Received: May 18, 2021; Accepted: June 28, 2021; Published: July 28, 2021

\begin{abstract}
We present the process of development and implementation of an internal audit for prevention of spread of COVID-19 at a tertiary eye hospital, which can be used for further improvement of the system and be utilized by similar organizations to enhance patient and staff safety and quality of service. It is a Single center prospective audit. Based on the guidelines put forth by various international (Centers for Disease Control and Prevention, World Health Organization) and Indian (Ministry of Health and Family Welfare, All India Ophthalmological Society) authorities, preventive protocols were formulated. To ensure compliance of these protocols, audit sheets in the form of checklists and questionnaires were prepared. Facility and process audits were undertaken for registration, outpatient department and pre-surgical counseling. Pilot audits were carried out to develop more detailed audits, and implemented for one week. There was $100 \%$ compliance from the staff in personal protective equipment usage and hand hygiene. However, 21 incidents of physical distancing not being maintained by them were noted. Though there was $100 \%$ compliance in use of facemask by the staff and patients, $34.5 \%$ of patients were noticed wearing the mask improperly. It was also noted that, at the entry, thermal screening was not performed at $6 / 53(11.3 \%)$ observations with no thermal screener at $3 / 23$ instances. This study describes the process of formulating and conducting audits as a monitoring and evaluation tool, which is most relevant in ongoing COVID-19 pandemic to ensure safety of patients and health care workers.
\end{abstract}

Keywords: COVID-19, Audit, Eye Hospital, Monitoring, Evaluation

\section{Introduction}

SARS-CoV-2 favorably known as COVID 19 was declared a pandemic by WHO in March 2020. [1] As already established, initial cases likely occurred via zoonotic transmission in Wuhan region of China in December 2019. [2] With an R0 factor (average number of people that one infected person will pass the virus to) ranging from 2 to 6.6 , this virus shows rapid human-tohuman transmission. [3] Further, the fact that the disease has an elusive nature with an asymptomatic carrier state of around $40 \%$ makes it even more dangerous.

Various protocols and preventive strategies have been devised by international authorities [4-7] all over the world to prevent the spread of this virus in the general population as well as to the health care workers. Various governments all over the world have issued a series of guidelines primarily focusing on protection of all concerned and prevention of the spread of Infection. 
Since no pharmaceutical drug has shown promise till now, [8] we need to stringently follow these preventive protocols on isolation, social distancing, hand hygiene, mask wearing, fomite disinfection as the "new normal", till either a vaccine becomes available or herd immunity develops.

So, the need of the hour remains to be able to improve the compliance rate for these protocols which will be extremely important in decreasing the transmission of the virus and thus, ensuring long term safety of the employees and patients. Continuous training and retraining will likely ensure the compliance of these guidelines, however, developing a strategy in the form of checklists and questionnaires (i.e. audit) to monitor these guidelines is of utmost importance.

Internal audit is a systematic evaluation of the quality system of a hospital which aims to improve patient safety by measuring performance of healthcare providers and preconditions for safe care and compares these outcomes with (national) standards and guidelines. The measurements are performed by an audit team comprising of internal peers (i.e. employees of a hospital who audit colleagues of other departments) [9].

The audit cycle comprises of four essential steps- "Plan Do- Check- Act" which effectively means that an audit must consist of a topic, appropriate practice standards, observation and testing against the selected standards, followed by identifying the areas for improvement, and subsequent interventions and demonstration of improvement in practice. The last point implies closing the audit loop by re-training the staff in areas of poor compliance and then re-auditing to identify the improvement.

Literature suggests that the compliance rate of the staff on infection control audits ranges from $28 \%$ to $100 \%$ depending on the tool assessed [9-12].

Cajuan $\mathrm{Xu}$ et al conducted daily audits for 3 days in January 2020 in a general tertiary care hospital in Zhejiang, China and found that the rate of proper mask wearing by staff was only $73 \%$ and hand hygiene compliance was as low as $40 \%$. [10] Similar compliance rate in hand hygiene and around $50 \%$ compliance in use of protective equipment was reported in another study, further highlighting the importance of conducting audits on a regular basis. [11] Bryce et al conducted an audit on the infection control knowledge assessment and found a poor response in routine precautions, personal protection for tuberculosis and hand hygiene practices $(47 \%, 49 \%$ and $68 \%$ respectively) [12].

The purpose of our study is to present the process of development and implementation of an audit mechanism for prevention of spread of COVID in an eye care hospital so that the learnings can be used for further improvement of our system and also by similar organizations to enhance patient and staff safety and quality of services.

\section{Methods}

India saw one of the strictest lockdowns in the whole world, with the first phase lasting 21 days, from 25 March till 14 April 2020. Our hospital is a tertiary eye care institute in Delhi, India catering to all specialties of ophthalmology and has a daily patient footfall of more than 800 patients. We were one of the first eye hospitals in India to resume services after the lockdown, and the work was increased in graded manner. In the initial phase of the crisis, when we started seeing patients again, we realised that there were no official guidelines pertaining to eye care institutes. We started framing our guidelines using information from governmental sources and some expert recommendations, extrapolating to our setting. Initially the sources included guidelines from Ministry of Health \& Family welfare, [6] Indian Council of Medical Research, [13] Medical council of India for telemedicine [14] and state government guidelines. Subsequently, guidelines were modified according to the information available from American Academy of Ophthalmology, Centres for Disease Control and Prevention, All India Institute of Medical Sciences and publication in Indian Journal of Ophthalmology. All India Ophthalmic Society (AIOS) formed a body to bring out guidelines for clinical and community-based eye care for Indian practice.

As the nature of the pandemic and the accompanying learning has been very dynamic, multiple versions were developed. First version had the registration and out-patient department (OPD) management, personal protective equipment (PPE) and bio-medical waste guidelines. Subsequently, Operation theatre (OT) guidelines along with relevant housekeeping and bio medical waste elements were added. Third version updated the PPE guidelines along with adding the protocol for isolation of exposed staff, dealing with suspected patients and COVID testing in pre-operative patients. The final fourth version (appendix) also included guidelines for canteen and kitchen. At this stage, filling of an online form, declaring fitness was made mandatory for employees at the time of reporting.

A nodal body headed by the medical superintendent (MS) and the executive director (ED) was created for implementation of protocols. A nodal officer was appointed as the convenor and was trained in Indian Penal Code protocol and oriented with COVID-19 protocols and advisory. The body created was multi-disciplinary consisting of the CEO, MS, ED, ophthalmologists, anaesthetist, ocular pathologist, senior nurse, quality manager and human resource manager with clear cut designation of roles. All the cadres in the hospital were provided training. The trainings were held online by the nodal body members. Additionally, for the first month, daily reinforcement was carried out for the staff in the hospital, before starting the day.

Once the protocols were finalized, audit sheet was developed. The final version of the audit sheet and audit process was developed after the implementation of the protocols for the first month. The audit format included two broad categories, facility (including equipment) audit and process audits. The format was developed for all important work stations in the hospital. These included registration, OPD, OT, ward and pre-surgical counseling (PSC). Initial quick pilot audits $(\sim 1$ hour) were carried out at all the stations. The audit team consisted of the nodal team members and included medical, paramedical and non-medical 
personnel at the various stations. All team members were trained on the format and processes of the audit to cause minimum possible disruption in the routine functions. These facility and process audit sections consisted of answering the check-list using binary responses of yes or no. Observations regarding proportion or degree of compliance were not captured. They were carried out on alternate days in the first month of implementation with the help of 2 audit committee members, once in the morning at around 9.30 am and once at noon. Learnings were utilized for functional improvement and also to structure a detailed audit process. This detailed audit was implemented in the first week of June 2020 and each audit cycle included observation for a period of 2 hours at each station. The results were calculated as proportion of observations made and for some aspects on the basis of untoward incidents recorded at various stations within that time period. Additionally, a short feedback from process owners at each station, regarding any issues in implementation, was included. This was as per recommendations from some previously published studies on the importance of soft elements in audits. $[15,16]$

Data from the detailed audit was collected and entered into the previously developed excel sheet. For the equipment/ facility audit, adequacy was collated for each station and then for items which were common to various stations, that data was combined and analyzed for all the stations. For process audit, proportions were calculated for observations at each station separately and then collated for the same process throughout all stations.

\section{Results}

The observations of the audit team members included deficiencies and recommendation. These were categorized into two sections- facility audit and process audit. Some of these observations were utilized for improving the processes by taking corrective actions and some of the observations were used to strengthen the audit process, as shown in Table 1.

Table 1. Table describing the observations of the pilot audits with the corrective actions.

\begin{tabular}{|c|c|}
\hline OBSERVATIONS & CORRECTIVE ACTION \\
\hline \multicolumn{2}{|l|}{ FACILITY AUDIT } \\
\hline \multicolumn{2}{|l|}{ 1. Poster displaying hand wash and hand rub technique not present in the ward, } \\
\hline 3. There is no backup of thermal scanner available. & 4. Replacement process checked and checking of quantity of soap \\
\hline \multicolumn{2}{|l|}{$\begin{array}{l}\text { 4. Hand washing soap and sanitizers found to be in an } \\
\text { PROCESS AUDIT }\end{array}$} \\
\hline 2. Staff at registration sitting close to each other and using adjacent computer stations & 2. Distancing done after spacing out the stations. \\
\hline during rush hour. & 3. Instructed and this made as an element in the detailed audit. \\
\hline 3. One team member had his mask below nose. & 4. Marks made for proper distancing and these incidents to be \\
\hline $\begin{array}{l}\text { 4. In General Registration area and Pre-op Counselling patients/attendants are not } \\
\text { following the distancing properly. Similarly in semi-private OPD waiting area, marks } \\
\text { for patients standing in a queue for registration were not present on the floor. }\end{array}$ & $\begin{array}{l}\text { 5. Screeners checked for accuracy. Process of keeping them in } \\
\text { shade in between use were made. Presence of back-up }\end{array}$ \\
\hline 5. Thermal screening being done but scanners giving wrong reading on gate no. 1 . & screeners to be recorded in detailed audit. \\
\hline
\end{tabular}

Taking into account the deficiencies and recommendations, a detailed audit sheet was then developed (audit sheet available on request) and implemented for a period of 1 week.

\section{Results of the Detailed Audits}

At gate entry pointy, infrared thermometer was available with adequate battery supplies, although an additional thermometer wasn't available as back up thrice in 10 observations (30.0\%). Adequate soap and sanitizer were available. Sufficient number of self-declaration forms were present and a poster displaying hand wash and hygiene was present. 35 patients entering the hospital were observed. All of them were asked the questions regarding risk of exposure and temperature was recorded for all. Out of 55 patients observed at the hand washing station, $5(9.1 \%)$ patients did not follow the hand washing advice. All the staff members were wearing the PPE and mask as per the recommendations whereas 15 out of $59(20 \%)$ patients did not have their nose covered. Appropriate social distancing was not observed in 9 out of $59(15.2 \%)$ patients while entering and during 10 audit sessions of 2 hours each, there were 6 incidents of physical distancing not being maintained by staff while interacting.

At the registration section, all the seats were arranged at proper distance and there was adequate supply of soap and sanitizer. Poster displaying hand hygiene technique was properly displayed. However, the electronic pen being used was not being cleaned after each use in one observation period out of a total of 12 . Proper physical distancing was maintained with all the 40 patients observed. However, there were 2 incidents of staff not maintaining social distance during 10 audit sessions. All patients followed the one attendant policy. There was 1 incident of staff not posted at registration being in that area. All the 4 staff members observed were in proper PPE and out of 69 patients observed $9(13.1 \%)$ wore mask not covering their nose.

In the OPD, all slit-lamps had a barrier in place. There was adequate provision of soap and sanitizers with a poster depicting hand-hygiene properly displayed. There were 3 incidents out of $34(8.8 \%)$ observed when adequate physical 
distancing was not maintained between patient-doctor interaction. During 7 sessions of audit, there were 4 incidents of staff not maintaining physical distancing. All the staff was in proper PPE and patients wearing mask covering their noses. There was no patient with more than one attendant. There was no incident of improper hand hygiene by staff. At the end of the OPD, there were 2 incidents of bins overflowing with discarded PPE out of 16 observed (12.5\%).

At the PSC section, all the 42 patients observed were again asked possible risk factors for exposure. In one audit session out of 14, thermal screening was not being done due to absence of functioning screener. There were 2 incident of blood pressure cuff or ECG leads not being cleaned in between patients among 45 patients observed (4.4\%). There were 6 incidents of social distancing not being observed out of 75 patients observed $(6 \%) .9$ incidents of staff not maintaining social distancing were observed over 12 audit sessions. Proper hand hygiene by the staff was followed in all 37 patient interactions observed. All staff were found to be in proper PPE but 21 out of 69 (30.4\%) patients were observed wearing masks not covering their noses. 4 out of the 20
(20.0\%) bins observed were found to be overflowing.

In the in-patients ward, all the patients followed 1 attendant policy. There was no incident observed of improper PPE, inadequate hand hygiene or breach of proper physical distancing policy among 14 observations of staff and 16 of patients. Staff knew about the proper etiquettes for coughing and there was no incident of presence of staff not posted in the ward. Proper clinical notes were being maintained for each patients and there was a change in bed sheet with each patient. There was no incident of improper disposal of bio-medical waste.

In the OT, adequate PPE were found to be available and there was no incidence of any staff member not using proper PPE. There was no incidence of anyone not following hand hygiene among 27 personnel observed and no improper disposal of waste. Only the staff on duty was found in the OT on all days. Change between cases was observed and on all 7 days there was no incident of inadequate disinfection, not switching off of air conditioner or inadequate time gap observed as per the guidelines.

Table 2 depicts the audit results of common infection control measures for all stations combined.

Table 2. Consolidated audit results.

\begin{tabular}{lll}
\hline Item & Audit results & Remarks \\
\hline Availability of thermal screening devices & $20 / 23(86.9 \%)$ & Back up screeners absent in 3/10 observations at entry \\
Availability of soap/ hand sanitizer & $28 / 28(100 \%)$ & \\
Display of awareness messages & $26 / 29(89.6 \%)$ & \\
Thermal screening performed & $47 / 53(88.7 \%)$ & \\
Physical distancing not maintained with patients & $18 / 222(8.1 \%)$ & Maximum at gate entry point \\
Physical distancing not maintained among staff & 21 incidents & Maximum at Pre-surgical counselling section \\
Appropriate PPE worn by staff & $100 \%$ & \\
Mask not worn properly & $80 / 232(34.5 \%)$ & All by patients. Mostly not covering the nose. Maximum at Pre-surgical \\
Hand hygiene not followed by staff & No incident & 5/55 patients observed not following hand washing at entry
\end{tabular}

Feedback was also collected from the health personnel managing different stations (Table 3). Corrective actions were taken to improve the processes.

Table 3. Feedback by personnel.

\begin{tabular}{|c|c|c|}
\hline STATION & ISSUED RAISED BY PERSONNEL & CORRECTIVE ACTIONS \\
\hline Entry Gate & $\begin{array}{l}\text { "As we are giving form to patients to fill, there should be a table there for filling the forms". } \\
\text { "Temperature screener gives erroneous reading occasionally". }\end{array}$ & $\begin{array}{l}\text { A table organized there. } \\
\text { Back up screener arranged. Air-coolers } \\
\text { provided for moderating temperature. }\end{array}$ \\
\hline Registration & $\begin{array}{l}\text { "A bit longer time was being taken as it was sometimes difficult to listen to the patient } \\
\text { due to the introduced barriers and patients wearing masks". }\end{array}$ & $\begin{array}{l}\text { Noted and more time allotted for } \\
\text { registration. }\end{array}$ \\
\hline OPD & None & \\
\hline PSC & $\begin{array}{l}\text { "Room with chambers is small, so maintaining distance at PSC station in private OPD is } \\
\text { difficult sometimes as patients wait outside individual chambers within the room". }\end{array}$ & $\begin{array}{l}\text { Process of making patients wait outside the } \\
\text { main room and being called by turn to the } \\
\text { chamber was implemented. }\end{array}$ \\
\hline $\begin{array}{l}\text { IN-PATIENT } \\
\text { WARD } \\
\text { OT }\end{array}$ & $\begin{array}{l}\text { "In private rooms, gowns are being put up by staff on the sofas and chairs while taking a break } \\
\text { for lunch. Some hangers / place should be available to hang the gowns". } \\
\text { None }\end{array}$ & Hangers provided. \\
\hline
\end{tabular}

\section{Discussion}

The world is witnessing 'once-in-a-century' event in the form of the ongoing COVID-19 pandemic. Indian as well global medical fraternity has risen to the occasion in dealing with this global crisis. The initial steps of formulating guidelines by the AIOS have been bold, decisive and scientifically driven in their response to COVID-19. [7] As India and the world reopen after the lockdown and eye hospitals get ready to treat patients using these guidelines, the next step is to monitor the on-field use of these processes, which have been developed.

An established tool in healthcare for safety governance is clinical audit, which is essential in checking whether standards, protocols and regulations are being followed or not. [17] This is 
most relevant now, when hospitals are trying to implement the formulated policies to keep their health care workers and patients safe. Simple observations of our audit team helped us understand deficiencies, which would have been missed easily.

Posters increase knowledge and change attitudes and behavior. [18] Even though we knew posters showing importance of social distancing and hand washing techniques would be useful, it was only through pilot audit that their absence was pointed out in $10 \%$ places. Other important observations of the pilot audit was to check for back up thermal scanner and adequate hand wash, which may seem straightforward, but their absence can cause delays and disruption of normal hospital functioning. Other hospitals should also take note of these basic facility requirements, and have their audit teams monitor the same.

One of the most important areas in the hospital, which needs special attention, is the entry point. Dedicated teams and good housekeeping facilities for the entry point is important for safeguarding the patients and staff inside the hospital. Any red flags should be raised at the entry itself and corrective measures should be taken accordingly. At the hospital entrance, it was observed that $15.2 \%$ of the patients did not follow social distancing norms, however, $100 \%$ social distancing norms were followed at the registration section and $91.2 \%$ in the clinics. A 3-day audit from China has also shown $97 \%$ compliance to social distancing. ${ }^{[10]}$ This may be attributable to social awareness and its importance being highlighted repeatedly. Additionally, seating and queuing arrangements should be made keeping social distancing in mind.

Other basic requirement that has been emphasized is the use of facemasks. With most of the state governments making the use of facemasks mandatory, we observed $100 \%$ compliance of people using facemasks, as noted by other post COVID-19 audit studies conducted in China. [10] We however noted $34.5 \%$ of patients were wearing the mask with their nose exposed, and no incidence of improper usage of masks from the staff. The aforementioned study from China showed $26.21 \%$ of improper mask usage by staff, chances of which can be minimised through repeated training sessions.

Before resuming clinical activities, substantial amount of time was dedicated to training the medical staff and doctors in operations of the OPD and OTs, which included donning and doffing of PPE, wearing a face mask, coughing etiquettes and social distancing in the clinics. Supervisors in every department conducted practical one on one sessions and online didactic lectures for staff education. One positive outcome noted was the fact that there was $100 \%$ compliance from our health care staff in PPE usage and hand washing techniques. We would likewise urge other hospitals to conduct similar sessions for their staff and discuss any concerns with them before they start clinical service.

To close the loop of our audit, small feedback was also collected from the health personnel managing different stations. It was brought to the nodal team's notice that a table for the screening staff was missing at entry, along with hangers which were not available for staff to hang their gowns in the ward. Also, based on the staff feedback, registration time allotted per patient was increased to ensure smooth hospital functioning. These interventions helped us understand deficiencies from staff's perspective and corrective action was taken to minimize staff inconvenience and to avoid any untoward incident.

While conducting this audit and conducting literature search for the same, we found limited data on monitoring and evaluation implemented in eye hospitals post COVID- 19 pandemic. To the best of our knowledge, this is the first study to highlight the audit results of COVID-19 policies from India (PubMed search of keywords- COVID-19, India, eye, hospitals, audit). Other hospitals should also formulate their working policies and share their audit results for efficient handling of the disease outbreak. We also acknowledge our limitation of having presented results of a short one week audit and are in the process of conducting a more elaborate re-audit.

Every hospital is the same in its intent of service and yet may be different in the way it delivers healthcare. We encourage other hospitals to take ideas from our exercise and make their own audit forms, and monitor their functioning. Regular evaluations help make systems better, and also safeguard staff and patients from getting infected. ${ }^{[19]}$ Even though everyone should take responsibility and reasonable steps towards reducing risks of transmission, in the end, it is the establishment's common law obligation that their premises are open to public in a safe condition, without avoidable hazards [20].

In this hour of need, let eye hospitals lead the way in setting up safe workable systems and evaluating on-field protocols for other hospitals and specialties to follow.

\section{Conclusion}

Audits are a well-known and effective way of keeping in check the stringent implementation of the made protocols. We conducted an internal audit to ensure the following of protocols to prevent and minimize the spread of COVID 19 among patients and healthcare staff. The results indicated various areas where the instructions were not being followed, and measures were taken to further implement them. Also, proper mask wearing and hand hygiene practices were documented. Furthermore, to close the audit loop, proper feedback was taken from the staff and corresponding measures advocated.

\section{Acknowledgements}

The authors deeply thank the members of our COVID-19 nodal and audit team for their help in preparing and implementing the hospital guidelines and for carrying out the audit with great sincerity and dedication.

\section{References}

[1] Available from: https://www.who.int/emergencies/diseases/novel-coronavirus2019. [Last accessed on 2020 May 27]. 
[2] Mackenzie JS, Smith DW. COVID-19: a novel zoonotic disease caused by a coronavirus from China: what we know and what we don't. Microbiol Aust. 2020; MA20013. doi: 10.1071/MA20013.

[3] Available from: https://www.bbc.com/news/health-52473523 [Last accessed on 2020 May 27].

[4] Occupational Safety and Health Administration. Guidance on Preparing Workplaces for COVID-19. Available at https://www.osha.gov/Publications/OSHA3990.pdf. Accessed: May 27, 2020.

[5] Available from: https://www.who.int/emergencies/diseases/novelcoronavirus-2019/technical-guidance/infection-prevention-andcontrol [last accessed 2020 May 27].

[6] Available from: https://www.mohfw.gov.in/. [Last accessed on 2020 May 28].

[7] Available from: https://aios.org/pdf/AIOS-OperationalGuidelines-COVID19.pdf [last accessed 2020 May 27].

[8] Available from: https://www.cdc.gov/coronavirus/2019ncov/hcp/therapeutic-options.html.

[9] Van Gelderen SC, Zegers M, Boeijen W, et al. Evaluation of the organisation and effectiveness of internal audits to govern patient safety in hospitals: a mixed methods study. BMJ Open 2017; 7: e015506.

[10] Xu C, Jin J, Song J, et al. Application of refined management in the prevention and control of coronavirus disease 2019 epidemic in non-isolated areas of a general hospital. Int J Nurs Sci. 2020; 7 (2): 143-147.

[11] Flanagan P. Current standards for infection control: audit assures compliance. Br J Nurs. 2009; 18 (16): 970-975.
[12] Bryce EA, Scharf S, Walker M, Walsh A. The infection control audit: the standardized audit as a tool for change. Am J Infect Control. 2007; 35 (4): 271-283.

[13] Available from: https://www.icmr.gov.in/pdf/covid/techdoc/EC_Guidance_CO VID19 06052020.pdf

[14] Available from: https://www.mohfw.gov.in/pdf/Telemedicine.pdf

[15] Turner CM, Bentley TM, Crampton JA, Rahman S. Making audit sexy. Consultants do sexy audit. BMJ. 2010; 340: c2642.

[16] Van Gelderen SC, Zegers M, Robben PB, Boeijen W, Westert GP, Wollersheim HC. Important factors for effective patient safety governance auditing: a questionnaire survey. BMC Health Serv Res. 2018; 18 (1): 798

[17] Hanskamp-Sebregts M, Zegers M, Boeijen W, Westert GP, van GurpPJ, Wollersheim H. Effects of auditing patient safety in hospital care: design of a mixed-method evaluation. BMC Health Serv Res 2013; 13 (1): 226.

[18] Berg, J. Creating a professional poster presentation: focus on nurse practitioners. Journal of the American Academy of Nurse Practitioners 2005, 17, 245- 248.

[19] Barnes M, Sax PE. Challenges of "Return to Work" in an Ongoing Pandemic [published online ahead of print, 2020 Jun 18]. N Engl J Med. 2020.

[20] Available from: https://www.natlawreview.com/article/reopening-economy-and-getting-back-to-business-businessowners-liability-risk-when. 